\title{
QUESTIONÁRIO: CONHECIMENTOS BÁSICOS DA POPULAÇÃO SOBRE HOMEOPATIA
}

\author{
CATEGORIA: SAÚDE COLETIVA/ EPIDEMIOLOGIA
}

CENTRO UNIVERSITÁRIO SÃO CAMILO

\author{
Silva, L. L. ${ }^{1}$ \\ Germino, C.1 \\ Costa, N. B. 1 \\ Macedo, L. S. ${ }^{1}$ \\ Pecci, A. M. N. T. ${ }^{1}$ \\ Nery, T.C.S. ${ }^{2}$
}

${ }^{1}$ ACADÊMICOS DO CENTRO UNIVERSITÁRIO SÃO CAMILO - UNIDADE IPIRANGA ${ }^{2}$ DOCENTE DE "SAÚDE DO TRABALHADOR" - IASC- DO CENTRO UNIVERSITÁRIO SÃO CAMILO

Rua Tupiniquins, № 51, Jardim Bandeirantes - Ribeirão Pires - SP

CEP: 09440-010

(11) 97654-9893 e-mail: leandro.leal@aluno.saocamilo-sp.br ou leandroleal.silva.Ils@gmail.com 


\section{QUESTIONÁRIO: CONHECIMENTOS BÁSICOS DA POPULAÇÃO SOBRE HOMEOPATIA}

CATEGORIA: SAÚDE COLETIVA/ EPIDEMIOLOGIA

DESCRITORES: Homeopatia, Informação, População, Prática integrativa, Sistema Público de Saúde. 


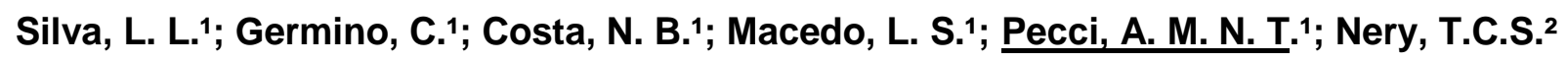

1 ACADÊMICOS DO CENTRO UNIVERSITÁRIO SÃO CAMILO - UNIDADE IPIRANGA

${ }^{2}$ DOCENTE DE "SAÚDE DO TRABALHADOR" - IASC VII - DO CENTRO UNIVERSITÁRIO SÃO CAMILO

\section{RESUMO:}

INTRODUÇÃO: A Homeopatia é reconhecida como especialidade médica no Brasil desde meados de 1980, porém, somente a partir de 2006 fora assegurada aos usuários do Sistema Único de Saúde (SUS). Essa terapia de ação generalista foi introduzida com o escopo de realocar o indivíduo no centro da atenção, compreendendo-o em seus aspectos biopsicossociais, contribuindo para a relação médico-paciente e humanização do atendimento. Todavia, os estudos demonstram que o conhecimento da população e profissionais da saúde, incluindo acadêmicos, é superficial e incoerente, visto que as informações sobre a prática não condizem com a realidade médica provavelmente devido a matérias de caráter depreciativo veiculadas pela mídia, que é a principal fonte de divulgação sobre a Homeopatia.

OBJETIVOS: Alcançar a dimensão do conhecimento populacional acerca do tema e identificar o quanto este se coaduna com a realidade da área.

METODOLOGIA: Através de pesquisa descritiva, foi aplicado questionário adaptado, com questões objetivas, em uma amostra de 85 indivíduos participantes de um evento beneficente na cidade de Guarulhos-SP. Entregue panfleto informativo acerca do tema após a entrevista. O trabalho foi realizado de acordo com a Resolução 510 de 2016. Os dados obtidos foram analisados por frequência simples, porcentagem, teste exato de Fisher e teste do Qui Quadrado. Foram utilizados os softwares IBM SPSS Statistics Subscription e Microsoft Excel Office 2016.

RESULTADOS: Os resultados demonstram que $72 \%$ já ouviram falar em Homeopatia, sendo que $84,7 \%$ nunca realizaram tratamento homeopático. Cerca de $40 \%$ obtiveram conhecimento através de livros, jornais, revistas e televisão, 16,5\% através de médicos e 4,7\% em farmácias. Temos que $47,5 \%$ acham que os medicamentos são preparados com produtos derivados exclusivamente de plantas e 5\% pensam que são agulhas. Na amostra, $75,4 \%$ acertou que é uma especialidade médica, mas 4\% pensam que é terapia com psicólogo e $18 \%$ não sabem o que é Homeopatia. Em paralelo, $62 \%$ sabem que são tratadas doenças de causas físicas e emocionais.

CONCLUSÃO: Em análise, apesar de grande parte da amostra ter acesso às informações, o conhecimento advém de fatos cotidianos e é superficial, fomentando conclusões incorretas acerca da Homeopatia; em suma, faz-se indispensável maior divulgação de instruções fidedignas e informações de qualidade à população, profissionais e acadêmicos da saúde, afim de propagar o esclarecimento populacional acerca desta especialidade médica bissecular. 
DESCRITORES: Homeopatia, Informação, População, Prática integrativa, Sistema Público de Saúde.

\section{QUESTIONÁRIO: CONHECIMENTOS BÁSICOS DA POPULAÇÃO SOBRE HOMEOPATIA}

\section{INTRODUÇÃO}

A Homeopatia se originou na Alemanha no final do século XVIII, com o médico Chistian Frederique Samuel Hahnemann. [1,2] Seus princípios incluem: “lei dos semelhantes”, em que a substância deve produzir, no homem são, sintomas semelhantes aos da doença que cura; a "experimentação no homem são"; e o "uso de doses mínimas", as quais são diluídas em solventes, como água ou álcool. [1]

Hahnemann traduziu o Tratado de Matéria Médica do inglês William Cullen, em 1790, quando teve contato com a China officinalis (quinina) e suas propriedades curativas contra a malária; em uma época de experimentos científicos acerca dos efeitos causados pela ingestão de substâncias em indivíduos sadios, o médico testou em si mesmo os efeitos da quinina, desenvolvendo quadro clínico semelhante à malária. [3] Assim, percebeu que a droga acarretava os mesmos sintomas da doença que ela mesma curava, e em outra perspectiva, curava uma doença cujos sintomas ela própria poderia desencadear, dando origem ao princípio Similia similibus curantur ou "semelhante cura semelhante" - batizando a Homeopatia, do grego homoion similar e pathos doença. [3]

As drogas eram testadas em dose tóxica, hipotóxica e dinamizada, em indivíduos sadios, sendo observados e compilados todos os sintomas físicos, emocionais e mentais do experimentador; as altas doses provocavam quadros de intoxicação, portanto Hahnemann desenvolveu o método de diluição em água e álcool, associado ao processo de dinamização, em que a diluição progressiva é associada à sucussão do preparado, configurando o princípio da "dose mínima". [3,4]

A Homeopatia chegou ao Brasil somente em meados de 1840; todavia, foi reconhecida como especialidade médica na década de 1980 pela Associação Médica Brasileira e Conselho Federal de Medicina. [1,2] É uma opção de cuidado recomendada pela Organização Mundial de Saúde (OMS), que divulgou documento incentivando a integração dessa e outras medicinas tradicionais aos sistemas nacionais de saúde [5]. Atualmente no Brasil existem cerca de 15 mil médicos homeopatas, sendo um dos países com maior número de profissionais no mundo; estima-se que 17 milhões de brasileiros já recorreram ao uso da Homeopatia. [3]

Apesar de ser uma das práticas integrativas no Sistema Único de Saúde (SUS), de acordo com a Portaria 971 - que aprovou a Política Nacional de Práticas Integrativas e Complementares (PNPIC) e assegura o acesso dos usuários do SUS à Homeopatia [7], essa especialidade médica não é maciçamente aplicada nos serviços de saúde. Além disso, é pouco reconhecida entre acadêmicos e outros profissionais da área. [1,2,6] 
Partindo desse pressuposto, há o surgimento de questões acerca do esclarecimento que a população potencialmente usuária tem sobre Homeopatia e sua prática terapêutica. Por esse motivo, o presente trabalho levanta dados sobre o conhecimento geral sobre 0 tratamento homeopático, em uma amostra populacional de uma região carente da cidade de Guarulhos, no Estado de São Paulo; tal população participava de um evento beneficente local, onde foi oferecida atenção médica gratuita por alunos de Medicina do Centro Universitário São Camilo.

\section{OBJETIVOS}

O objetivo geral desta pesquisa é caracterizar o conhecimento da população sobre Homeopatia. Os objetivos específicos são: verificar se o conhecimento da população coincide com a realidade da especialidade médica e promover uma discussão a respeito dos dados obtidos.

\section{METODOLOGIA}

Este trabalho é resultado de uma pesquisa qualitativa descritiva, a qual apresenta dados do conhecimento da população estudada sobre a Homeopatia. Inicialmente foi realizado levantamento bibliográfico sobre a temática. Foi utilizado um questionário já validado na literatura, com questões objetivas (questionário adaptado) sobre dados demográficos e dados de conhecimento geral da população sobre Homeopatia (Anexo 1). O questionário foi aplicado em um evento beneficente, promovido pelo Curso de Medicina do Centro Universitário São Camilo juntamente com uma Instituição religiosa, em Guarulhos, São Paulo no dia 12/10/2017. A amostra conta com 85 indivíduos que estavam participando do evento e não foi selecionada por cor, raça, orientação sexual, sendo os participantes indivíduos que estavam à espera do atendimento. Os critérios de inclusão considerados foram: maiores de 18 anos e questionários devidamente preenchidos.

Os cinco alunos membros da Liga Acadêmica de Homeopatia da São Camilo (LAHOSC) tiveram a autorização por parte dos organizadores para aplicar o questionário no evento, os quais foram devidamente orientados de modo a realizar a correta abordagem ao participante, com o objetivo de minimizar o viés da pesquisa.

Os indivíduos que concordaram em participar da pesquisa assinaram o Termo de Consentimento livre e Esclarecido (TCLE).

O trabalho foi realizado de acordo com a RESOLUÇÃO № 510, de 07 de abril de 2016, do Ministério da Saúde, a qual trata sobre normas aplicáveis à pesquisa em Ciências Humanas e Sociais, em trabalhos que lidam com informações obtidas diretamente com o participante da pesquisa, quanto à não avaliação e registro pelo sistema COEP.[8] 
"I - pesquisa de opinião pública com participantes não identificados;

IV - pesquisa censitária; e

VII - pesquisa que objetiva o aprofundamento teórico de situações que emergem espontânea e contingencialmente na prática profissional, desde que não revelem dados que possam identificar o sujeito"

Os dados obtidos foram analisados por frequência simples, porcentagem, teste exato de Fisher e teste do Qui Quadrado. Para isso foi utilizado o software IBM SPSS Statistics Subscription para a análise estatística e o Microsoft Excel Office 2016 para a organização dos dados.

Após a aplicação do questionário, foi entregue a cada participante um panfleto informativo contendo as orientações básicas sobre o tema. (Anexo 2)

\section{RESULTADOS}

Foram avaliados 85 questionários devidamente preenchidos.

I. Dados demográficos

Do total de participantes da pesquisa, 28 (32,9\%) foram indivíduos do sexo masculino, enquanto que $57(67,1 \%)$ foram do sexo feminino (Tabela 1$)$.

\section{Tabela 1: Sexo dos indivíduos da pesquisa}

e frequência (em \%)

\begin{tabular}{lrr} 
& Frequência & \multicolumn{1}{c}{ Porcentagem } \\
\hline Masculino & 28 & 32,9 \\
\hline Feminino & 57 & 67,1 \\
\hline Total & 85 & 100,0 \\
\hline
\end{tabular}

Em relação à escolaridade, pode-se perceber pela análise da Tabela 2 que a maioria dos participantes possui o ensino médio completo - 32 indivíduos $(37,6 \%)$ - e que 17 indivíduos entrevistados (20\%) tiveram acesso ao ensino superior.

Tabela 2: Escolaridade dos indivíduos da pesquisa e frequência (em \%)

\begin{tabular}{lrr} 
& Frequência & Porcentagem \\
\hline Fundamental Incompleto & 16 & 18,8 \\
\hline Fundamental Completo & 14 & 16,5 \\
\hline Médio Incompleto & 6 & 7,1 \\
\hline Médio Completo & 32 & 37,6 \\
\hline Universitário Incompleto & 6 & 7,1 \\
\hline Universitário Completo & 11 & 12,9 \\
\hline Total & 85 & 100,0 \\
\hline
\end{tabular}


Pode ser observado também que a maioria dos participantes possui mais de 50 anos - 31 indivíduos $(36,5 \%)$ - enquanto que apenas 4 (4,7\%) são menores de 20 anos (Tabela 3 ).

\section{Tabela 3: Faixa etária e frequência (em \%)}

\begin{tabular}{lrr} 
& Frequência & Porcentagem \\
\hline 18 a 20 anos & 4 & 4,7 \\
\hline 21 a 30 anos & 16 & 18,8 \\
\hline 31 a 40 anos & 13 & 15,3 \\
\hline 41 a 50 anos & 21 & 24,7 \\
\hline Acima de 50 anos & 31 & 36,5 \\
\hline Total & 85 & 100,0 \\
\hline
\end{tabular}

II. Dados referentes ao conhecimento da população acerca do tema

Sobre o conhecimento da população referente à área médica da Homeopatia, a maioria já "ouviu falar em Homeopatia" - totalizando 61 participantes da pesquisa (72\%), como pode ser observado no Gráfico 1.

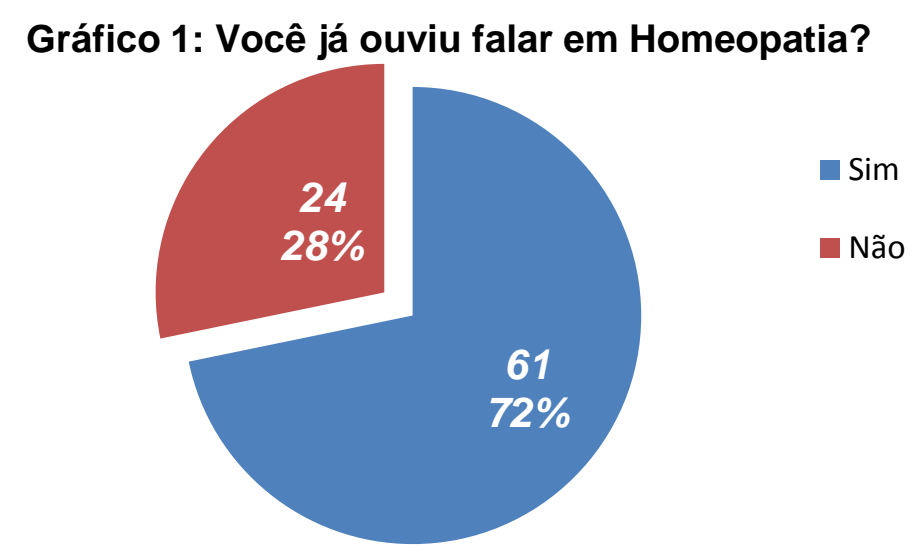

Esse dado foi fundamental à pesquisa, visto que o participante cuja resposta inicial fosse uma negativa, não saberia responder às perguntas posteriores do questionário aplicado, e em caso de tentativa de resposta às questões subsequentes, estas viriam a ser um viés na presente pesquisa; portanto, para as perguntas seguintes, esses indivíduos estão retratados como "Não tem conhecimento".

A maioria dos indivíduos afirmaram que obtiveram conhecimento sobre a Homeopatia através de algum veículo de mídia, como livro, jornais, revistas ou televisão - 34 indivíduos (40\%) - ou através de médicos - 14 indivíduos (16,5\%), conforme Tabela 4. 
Tabela 4: Conhecimento sobre Homeopatia através de:

\begin{tabular}{lr|r|} 
& Frequência & Porcentagem \\
\hline Não tem conhecimento & 24 & 28,2 \\
\hline Livro, jornais, revistas ou televisão & 34 & 40,0 \\
\hline Amigo, vizinho ou parente & 9 & 10,6 \\
\hline Médico & 14 & 16,5 \\
\hline Farmácia & 4 & 4,7 \\
\hline Total & 85 & 100,0 \\
\hline
\end{tabular}

Do total de participantes da pesquisa, apenas $13(15,3 \%)$ já realizaram algum tratamento com Homeopatia (Tabela 5).

Tabela 5: Já fez tratamento com Homeopatia?

\begin{tabular}{lrr} 
& Frequência & \multicolumn{1}{c}{ Porcentagem } \\
\hline Sim & 13 & 15,3 \\
\hline Não & 72 & 84,7 \\
\hline Total & 85 & 100,0 \\
\hline
\end{tabular}

Questionados sobre o que seria a Homeopatia (dentre as opções disponíveis), 46 indivíduos $54,1 \%$ do total de participantes e $75,4 \%$ dos indivíduos que afirmaram conhecer a Homeopatia afirmaram que a Homeopatia é uma especialidade da área da saúde que utiliza medicamentos para tratar doenças, enquanto que 11 indivíduos - 12,9\% do total de participantes e 18,0\% dos indivíduos que afirmaram conhecer a Homeopatia -assinalaram como opção a afirmativa "Não sei" para essa questão, mesmo afirmando conhecer a Homeopatia na primeira questão (Tabela 6).

Tabela 6: Para você, Homeopatia é:

Porcentagem absoluta quanto à afirmativa sobre o conhecimento Frequência Porcentagem da homeopatia $(n=61)$

\begin{tabular}{lccr}
\hline Sem conhecimento & 24 & 28,2 & - \\
\hline Não sei & 11 & 12,9 & 18,0 \\
\hline $\begin{array}{l}\text { Uma especialidade da área da saúde que } \\
\text { utiliza medicamentos para tratar doenças }\end{array}$ & 46 & 54,1 & 75,4 \\
\hline Terapia com psicólogo & & & 6,6 \\
\hline Total & 4 & 4,7 & 100,0 \\
\hline
\end{tabular}

Quanto ao questionamento sobre o que é utilizado no tratamento homeopático, 29 indivíduos $34,1 \%$ do total de participantes e $47,5 \%$ dos indivíduos que afirmaram conhecer a Homeopatia alegaram que são utilizados apenas produtos derivados de plantas, enquanto que $17-20,0 \%$ do total de participantes e $28,0 \%$ dos indivíduos que afirmaram conhecer a Homeopatia - afirmaram que são utilizados medicamentos preparados em glóbulos e gotas (Tabela 7). 
Tabela 7: No tratamento homeopático são utilizados:

\begin{tabular}{lrrr} 
& Frequência & Porcentagem & $\begin{array}{c}\text { Porcentagem absoluta quanto à afirmativa sobre o } \\
\text { conhecimento da homeopatia }(\mathrm{n}=61)\end{array}$ \\
\hline Sem conhecimento & 24 & 28,2 & - \\
\hline Não sei & 12 & 14,1 & 19,6 \\
\hline $\begin{array}{l}\text { Apenas produtos } \\
\text { derivados de plantas. }\end{array}$ & 29 & 34,1 & 47,5 \\
Medicamentos preparados & 17 & 20,0 & 28,0 \\
em glóbulos e gotas & & & 4,9 \\
\hline Agulhas & 3 & 3,5 & 100 \\
\hline Total & 85 & 100,0 & \\
\hline
\end{tabular}

A questão referente ao cuidado do especialista homeopata apresentou como principal resposta as "doenças físicas e de causa emocional, tratando o indivíduo como um todo" (38 indivíduos - 44,7\% do total de participantes e $62,2 \%$ dos indivíduos que afirmaram conhecer a Homeopatia); todavia, foi considerável o número de participantes a declarar "Não sei" como resposta, sendo 15 indivíduos - $17,6 \%$ do total de participantes e $24,6 \%$ dos indivíduos que afirmaram conhecer a homeopatia, conforme análise da Tabela 8.

Tabela 8: O Médico homeopata é o especialista que cuida de:

Porcentagem absoluta quanto à afirmativa sobre o conhecimento da homeopatia $(n=61)$

\begin{tabular}{lrrr} 
& Frequência & Porcentagem & \\
\hline Sem conhecimento & 24 & 28,2 & $-24,6$ \\
\hline Não sei & 15 & 17,6 & 62,2 \\
\hline $\begin{array}{l}\text { Doenças físicas e de causa emocional, } \\
\text { tratando o indivíduo como um todo. }\end{array}$ & 38 & 44,7 & 9,8 \\
\hline Apenas doenças do sangue & 6 & 7,1 & 3,4 \\
\hline Apenas doenças alérgicas & 2 & 2,4 & 100,0 \\
\hline Total & 85 & 100,0 & \\
\hline
\end{tabular}

\section{DISCUSSÃO}

Apesar da Homeopatia ser uma especialidade médica reconhecida no país há mais de 30 anos, e desde 2006 assegurada aos usuários do Sistema Único de Saúde (SUS) [1], seu acesso ainda é questionável, visto que grande número de indivíduos ainda não possui conhecimento sobre a área.

A Homeopatia foi introduzida no sistema de saúde com o intuito de realocar o indivíduo no centro da atenção da saúde, compreendendo-o em diferentes aspectos, de modo a contribuir na relação médico-paciente e humanizando o atendimento, como um dos princípios de reduzir as intervenções e contribuindo para a qualidade de vida e uso racional de medicamentos; todavia, diversas 
pesquisas ainda demonstram a baixa adesão e conhecimento da população e outras áreas médicas quanto à Homeopatia [9], dado que é semelhante ao obtido no presente trabalho.

Dos 85 participantes, 61 deles (72\%) afirmaram já ter ouvido falar em Homeopatia, enquanto que no estudo de Furukawa, Morikawa e Gutiérrez [10], autores do questionário utilizado de modo adaptado na atual pesquisa, 92\% dos participantes responderam "sim" a essa mesma pergunta. Um fato que deve ser considerado é que a atual pesquisa foi realizada em um evento frequentado por indivíduos cujo objetivo era obter atendimento médico, enquanto que no estudo de Furukawa, foi no interior de uma farmácia de manipulação, podendo criar um viés na seleção dos pacientes. Em comparação ao estudo de Oliveira e Salvi [2], apenas 3,4\% não sabiam o que era a Homeopatia; entretanto, vale ressaltar que neste estudo há um grupo selecionado de 120 estudantes de farmácia, fato que pode ser considerável - pois envolve uma população com conhecimento acadêmico relacionado à área de saúde, o que pode contribuir para o número baixo de desconhecimento sobre a Homeopatia.

As tabelas 9, 10 e 11 demonstram o conhecimento da Homeopatia segundo sexo, escolaridade e faixa etária, respectivamente, e demonstram que grande número de indivíduos da pesquisa possui conhecimento sobre a homeopatia, no entanto, também é considerável o número dos que desconhecem.

\begin{tabular}{|c|c|c|c|c|}
\hline \multicolumn{5}{|c|}{$\begin{array}{l}\text { pesquisa e o conhecimento em Homeopatia (e em \%) } \\
\text { Vocé já ouviu falar em } \\
\text { Homeopatia? }\end{array}$} \\
\hline & & \multirow{2}{*}{$\operatorname{Sim}(21,18)$} & \multirow{2}{*}{ Não } & \multirow{2}{*}{$\frac{\text { Total }}{28(32,95)}$} \\
\hline Sexo & Masculino & & & \\
\hline & Feminino & $43(50,58)$ & $14(16,47)$ & $57(67,05)$ \\
\hline Total & & $61(71,76)$ & $24(28,24)$ & $85(100,0)$ \\
\hline
\end{tabular}

Conforme análise da Tabela 9 pode-se perceber que apesar de o número de mulheres que conhecem a Homeopatia ser maior, se comparado ao número de homens que conhecem tal área médica, com o resultado do Teste Exato de Fisher de 0,31 ( $p>0,05)$ não há predileção entre os sexos e o conhecimento sobre a Homeopatia, pois o p é insignificante.

A Tabela 10 apresenta a escolaridade de acordo com o conhecimento sobre a Homeopatia, e de acordo com o resultado do Teste do Qui Quadrado de 0,52 ( $p>0,05)$ pode-se afirmar que não há relação entre tais dados. 
Tabela 10: Relação entre escolaridade e conhecimento sobre Homeopatia (e em \%)

\begin{tabular}{llrr|r}
\multicolumn{2}{l}{ Dados analisados } & \multicolumn{2}{c}{$\begin{array}{c}\text { Você já ouviu falar em } \\
\text { Homeopatia? }\end{array}$} & \\
\hline \multirow{2}{*}{ Escolaridade } & \multicolumn{1}{c}{ Sim } & \multicolumn{1}{c}{ Não } & \multicolumn{1}{c}{ Total } \\
\cline { 2 - 5 } & Fundamental incompleto & $9(10,5)$ & $7(8,2)$ & $16(18,7)$ \\
\cline { 2 - 5 } & Fundamental Completo & $10(11,8)$ & $4(4,7)$ & $14(16,5)$ \\
\cline { 2 - 5 } & Médio incompleto & $4(4,8)$ & $2(2,4)$ & $6(7,2)$ \\
\cline { 2 - 5 } & Médio Completo & $24(28,2)$ & $8(9,4)$ & $32(37,6)$ \\
\cline { 2 - 5 } & Universitário incompleto & $4(4,8)$ & $2(2,4)$ & $6(7,2)$ \\
\cline { 2 - 5 } & Universitário Completo & $10(11,8)$ & $1(1,2)$ & $11(13,0)$ \\
\hline Total & $61(71,7)$ & $24(28,3)$ & $85(100,0)$ \\
\hline
\end{tabular}

Do mesmo modo, o Teste do Qui Quadrado de 0,52 ( $p>0,05)$ confirma que também não há relação entre faixa etária e conhecimento sobre homeopatia (Tabela 11).

\section{Tabela 11: Relação entre faixa etária e conhecimento sobre Homeopatia} (e em \%)

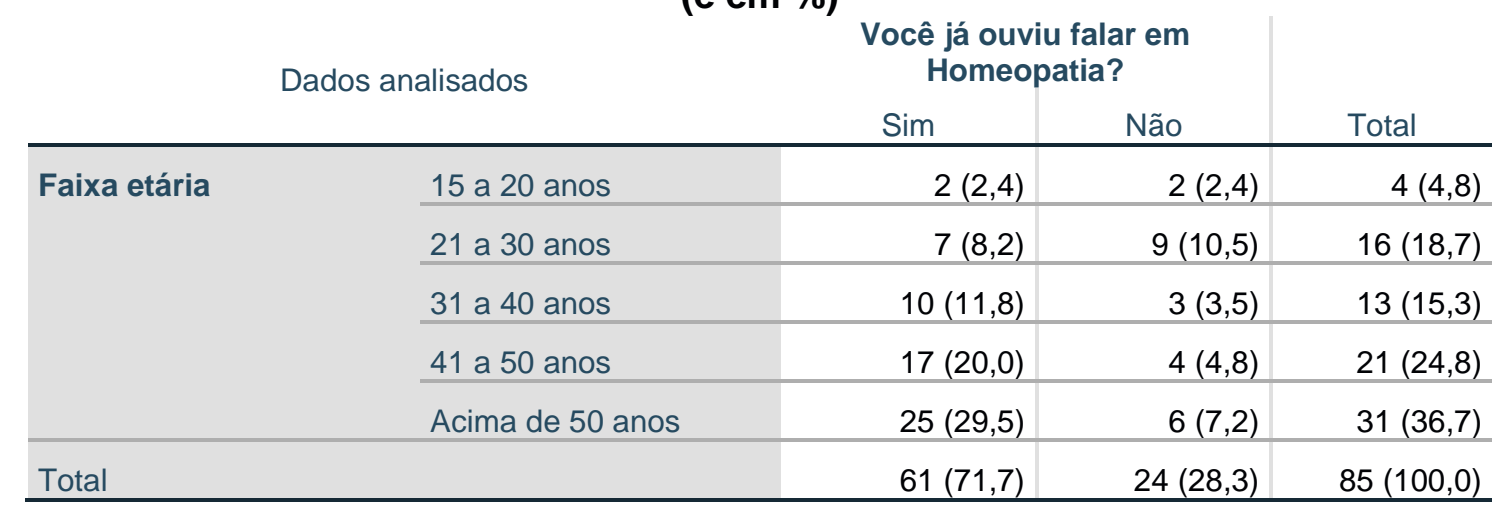

Em relação ao tratamento homeopático, no atual estudo apenas 15,3\% das pessoas (TABELA 5) já realizaram tratamento com Homeopatia, enquanto que no estudo de Freitas e Almeida [1] 26\% dos entrevistados relataram ter usado, sendo relativamente semelhante o baixo índice de uso do tratamento homeopático. Já no estudo de Furukawa, Morikawa e Gutiérrez [10], 50\% das pessoas já tinham utilizado tal tratamento e $44,4 \%$ dos participantes do estudo de Oliveira e Salvi [2] já haviam usado alguma composição, demonstrando um número admiravelmente maior.

Do conhecimento sobre Homeopatia (TABELA 4), apenas 18 indivíduos obtiveram conhecimento através de um médico ou farmácia, totalizando 14 (16,5\%) e 4 indivíduos (4,7\%) respectivamente, sendo um dado parecido ao obtido pelo estudo de Furukawa, Morikawa e Gutiérrez [10], em que $25 \%$ dos entrevistados haviam conhecido a Homeopatia através destes mesmos meios. No estudo descrito por Oliveira e Salvi [2], foi maior o número de participantes que conheceram a área através de livros, jornais, revistas e televisão, sendo afirmado por $43,3 \%$ dos participantes, de modo semelhante ao presente estudo, cujo conhecimento por esses meios foi afirmado por $40,0 \%$ dos 
entrevistados; no entanto, diferiu relativamente quanto ao conhecimento através de amigo, vizinho ou parentes, visto que no estudo de Oliveira e Salvi [2] foi de 33,3\% e aqui de apenas 10,6\%.

No estudo de Fortunato e Almeida [1], realizado com acadêmicos de Farmácia, aproximadamente $38 \%$ dos participantes afirmaram conhecer a Homeopatia através de "pessoas conhecidas", 18\% através de lojas de produtos naturais e 14\% através da formação acadêmica e da literatura.

Já no estudo de Külkamp IC. apud Souza Junior et. Al. [5], no qual foram entrevistados acadêmicos do curso de Medicina da UNISUL de Tubarão - SC, 92,9\% dos participantes afirmaram conhecer a Homeopatia e a maioria obteve o conhecimento através de propagandas de TV, outros profissionais e outras fontes não esclarecidas, apesar de serem acadêmicos da área da saúde, fato que também deve ser considerado para avaliar o alto número de cientes sobre a homeopatia - vale ressaltar que apesar da Homeopatia ser reconhecida como especialidade médica na década de 1980, ainda há certo estigma tanto pela população [1] quanto por médicos, acadêmicos e outros profissionais da saúde [9,11-14]. Um dado a ser considerado, é que pouco se comenta na área da saúde, ou seja, pouco se difunde a informação, refletindo também no maior conhecimento da população entrevistada através de veículos de mídia "Livros, jornais, revistas ou televisão", em que 34 indivíduos (40\%) responderam ter obtido conhecimento dessa maneira.

Desse modo, a Tabela 12 apresenta a via de contato com Homeopatia e o que é a Homeopatia para os participantes da pesquisa, a fim de relacionar se o modo como o paciente conheceu a especialidade lhe apresentou dados concretos e condizentes sobre tal área médica, e apesar das diversas formas de conhecimento, não houve unanimidade sobre a resposta quanto ao que é a homeopatia. Dos indivíduos que afirmaram conhecer a Homeopatia através de livro, jornais, revistas ou televisão, 8 (9,4\%) afirmaram não saber o que era a Homeopatia, enquanto que 25 (29,4\%) afirmaram que é "uma especialidade da área da saúde que utiliza medicamentos para tratar doenças", representando o maior número de respostas; todavia, ainda houve resposta como "terapia com psicólogo", de modo semelhante quando afirmado que o conhecimento ocorreu através de médico.

Esse dado permite comparar a uma análise relatada no estudo de Külkamp IC. apud Souza Junior et. Al. [5], onde o autor afirma que o conhecimento é advindo de fatos cotidianos e que as ideias sobre o assunto são superficiais; em paralelo, pode-se questionar se a instrução obtida por profissionais da saúde é válida, e se os veículos midiáticos propagam tais informações com credibilidade. 
Tabela 12: Relação entre conhecimento sobre homeopatia e o que é a homeopatia (e em \%)

\begin{tabular}{llrr|r|r} 
& & & \multicolumn{2}{c}{ Para você, Homeopatia é: } \\
& Dados analisados & Não sei & $\begin{array}{c}\text { Uma especialidade } \\
\text { da área da saúde... }\end{array}$ & \multicolumn{1}{c}{$\begin{array}{c}\text { Terapia com } \\
\text { psicólogo }\end{array}$} & \multicolumn{1}{c}{ Total } \\
\hline Conhecimento & Não tem conhecimento & $24(28,2)$ & $0(0,0)$ & $0(0,0)$ & $24(28,2)$ \\
\cline { 2 - 6 } $\begin{array}{l}\text { sobre } \\
\text { Homeopatia } \\
\text { através de: }\end{array}$ & Livro, jornais, revistas ou televisão & $8(9,4)$ & $25(29,4)$ & $1(1,2)$ & $34(40,0)$ \\
\cline { 2 - 6 } & Amigo, vizinho ou parente & $3(3,5)$ & $5(5,9)$ & $1(1,2)$ & $9(10,6)$ \\
\cline { 2 - 6 } & Médico & $0(0,0)$ & $12(14,1)$ & $2(2,4)$ & $14(16,5)$ \\
\hline Total & & $0(0,0)$ & $4(4,8)$ & $0(0,0)$ & $4(4,7)$ \\
\hline
\end{tabular}

A fim de relacionar a faixa etária e o conhecimento sobre a Homeopatia, a Tabela 13 demonstra que não há relação propriamente dita entre idade e conhecimento, de modo semelhante ao retratado pela Tabela 11; no entanto, apesar de não ter essa relação estatisticamente comprovada, pode-se ter uma livre aquiescência de que os indivíduos de maior idade (acima de 41 anos) sabem sobre a área quando afirmaram em questão anterior. Não foram encontrados dados na literatura para comparar conhecimento sobre a Homeopatia e a faixa etária; porém, pode ser questionado se o fato de indivíduos de idade mais avançada terem tal conhecimento seja devido à maior incidência de doenças nessa faixa etária, e, assim, maior busca por tratamentos médicos.

Tabela 13: Relação entre Faixa etária e o que é a homeopatia

\begin{tabular}{|c|c|c|c|c|c|c|}
\hline & & $\begin{array}{c}\text { Sem } \\
\text { conhecimento }\end{array}$ & $\begin{array}{l}\text { Para você, } \\
\text { Não sei }\end{array}$ & $\begin{array}{l}\text { Homeopatia é: } \\
\text { Uma especialidade } \\
\text { da área da saúde... }\end{array}$ & $\begin{array}{c}\text { Terapia com } \\
\text { psicólogo }\end{array}$ & Total \\
\hline \multirow[t]{5}{*}{ Faixa etária } & 15 a 20 anos & 2 & 1 & 1 & 0 & 4 \\
\hline & 21 a 30 anos & 9 & 1 & 6 & 0 & 16 \\
\hline & 31 a 40 anos & 3 & 2 & 6 & 2 & 13 \\
\hline & 41 a 50 anos & 4 & 1 & 15 & 1 & 21 \\
\hline & Acima de 50 anos & 6 & 6 & 18 & 1 & 31 \\
\hline Total & & 24 & 11 & 46 & 4 & 85 \\
\hline
\end{tabular}

Em relação ao o que é Homeopatia e o que é utilizado no tratamento homeopático (Tabela 14), pode-se constatar que muitas pessoas associam essa área médica à utilização de produtos derivados exclusivamente de plantas (29 indivíduos (34,1\%), sendo que $23(27,0 \%)$ consideram a Homeopatia como uma especialidade da área da saúde que utiliza medicamentos para tratar doenças. Resultados semelhantes foram encontrados e discutidos por Gecioni Loch-Neckel et al. [13]: em seu estudo com estudantes dos cursos de Farmácia, Medicina e Odontologia, 36,1\% dos participantes alegaram que a Homeopatia utiliza produtos naturais para o tratamento dos pacientes, resultado comparado com o estudo de Micali et al. (apud Gecioni Loch-Neckel et al. [13]) realizado em Vitória-ES, onde $38,4 \%$ da população entrevistada associou a Homeopatia a um tratamento 
natural. Os estudos afirmam que há associação da Homeopatia com tratamentos naturais à imagem de um medicamento não elaborado, sem a possibilidade de fornecer efeitos adversos.

Tabela 14: Relação entre o que é homeopatia e o que é utilizado no tratamento homeopático $(\mathrm{n}=61)$

\begin{tabular}{|c|c|c|c|c|c|c|}
\hline & & Não sei & $\begin{array}{c}\text { ratamento homeo } \\
\text { Apenas produtos } \\
\text { derivados de } \\
\text { plantas. }\end{array}$ & $\begin{array}{l}\text { ático são utiliza } \\
\text { Medicamentos } \\
\text { preparados... }\end{array}$ & Agulhas & Total \\
\hline \multirow{3}{*}{$\begin{array}{l}\text { Para você, } \\
\text { Homeopatia é: }\end{array}$} & Não sei & $5(5,9)$ & $4(4,7)$ & $1(1,2)$ & $1(1,2)$ & $11(12,9)$ \\
\hline & $\begin{array}{l}\text { Uma especialidade da } \\
\text { área da saúde... }\end{array}$ & $6(7,0)$ & $23(27,0)$ & $15(17,7)$ & $2(2,4)$ & $46(54,1)$ \\
\hline & Terapia com psicólogo & $1(1,2)$ & $2(2,4)$ & $1(1,2)$ & $0(0,0)$ & $4(4,7)$ \\
\hline \multicolumn{2}{|l|}{ Total } & $12(14,1)$ & $29(34,1)$ & $17(20,0)$ & $3(3,5)$ & $85(100,0)$ \\
\hline
\end{tabular}

A Homeopatia é uma terapêutica de ação generalista, todavia, muitos estudos demonstram que o conhecimento da população e de alguns profissionais da saúde, incluindo acadêmicos, associa o tratamento homeopático a doenças específicas, como em casos de doenças crônicas e doenças psicossomáticas. [10-14] No atual estudo pode-se considerar que também houve dúvidas sobre quais doenças ou fatores o especialista em Homeopatia é responsável, pois apenas 38 indivíduos $(44,7 \%)$ afirmaram que o homeopata "cuida de doenças físicas e de causa emocional, tratando o indivíduo como um todo", enquanto que $27,1 \%$ apresentou diferentes respostas entre "Não sei", "apenas doença do sangue" e "apenas doenças alérgicas" (Tabela 15), o que demonstra a necessidade de maiores conhecimentos a respeito dessa área médica.

\section{Tabela 15: Relação entre o que é homeopatia e o Médico homeopata}

\begin{tabular}{|c|c|c|c|c|c|c|}
\hline & & O Méd & $\begin{array}{l}\text { ico homeopata é o es } \\
\text { Doenças físicas e de } \\
\text { causa emocional, } \\
\text { tratando o indivíduo } \\
\text { como um todo. }\end{array}$ & $\begin{array}{l}\text { ecialista q } \\
\text { Apenas } \\
\text { doenças } \\
\text { do } \\
\text { sangue } \\
\end{array}$ & $\begin{array}{l}\text { e cuida de: } \\
\text { Apenas } \\
\text { doenças } \\
\text { alérgicas } \\
\end{array}$ & Total \\
\hline \multirow{3}{*}{$\begin{array}{l}\text { Para você, } \\
\text { Homeopatia é: }\end{array}$} & Não sei & $6(7,0)$ & $2(2,4)$ & $2(2,4)$ & $1(1,2)$ & $11(12,9)$ \\
\hline & $\begin{array}{l}\text { Uma especialidade } \\
\text { da área da saúde... }\end{array}$ & $9(10,6)$ & $32(37,7)$ & $4(4,7)$ & $1(1,2)$ & $46(54,1)$ \\
\hline & $\begin{array}{l}\text { Terapia com } \\
\text { psicólogo }\end{array}$ & $0(0,0)$ & $4(4,7)$ & $0(0,0)$ & $0(0,0)$ & $4(4,7)$ \\
\hline Total & & $15(17,7)$ & $38(44,7)$ & $6(7,0)$ & $2(2,4)$ & $85(100,0)$ \\
\hline
\end{tabular}

A Homeopatia é uma ciência com história bissecular, entretanto a desinformação da população é notável, segundo dados obtidos por esse trabalho; considerando que, na nossa amostragem, a maior fonte de informações é a mídia, tal desinformação pode ser oriunda de publicações e matérias 
de caráter depreciativo veiculados por revistas, redes sociais e televisão, visto que resultados favoráveis a essa especialidade médica raramente são mostrados ao público leigo. [15]

Também é questionável o motivo da amostragem do presente estudo, em sua maioria, já ter ouvido falar sobre Homeopatia, e, todavia, não ser adepta ao tratamento homeopático; tal realidade pode ter origens no preconceito existente no inconsciente coletivo, advindo das publicações depreciativas já mencionadas, as quais desconsideram os estudos científicos que validam o modelo homeopático - contribuindo para a manutenção de posicionamentos contrários a essa prática médica, e, portanto, perpetuando a desinformação e dificultando a adesão populacional à Homeopatia. [15]

\section{CONCLUSÃO}

É evidente que, mesmo que a população tenha acesso à área médica, as informações obtidas são restritas, logo o indivíduo acredita que conhece a Homeopatia, quando na verdade tem noção de modo muito superficial - não sabendo o que trata o especialista, como trata, e o diferencial entre essa medicina e medicinas tradicionais e/ou alopáticas, amplamente conhecidas.

Assim, mostra-se necessária uma maior divulgação acerca da Homeopatia para toda a população, incluindo profissionais da saúde e acadêmicos, a fim de aumentar o índice de esclarecimento populacional em relação ao tema - pois estes podem auxiliar no processo da divulgação da informação, bem como se beneficiar de tal terapia enquanto usuários.

\section{REFERÊNCIAS}

[1] Fortunato, Laise de Freitas; Almeida, Ana Flavia Santos Almeida Santos. Conhecimento sobre a homeopatia entre os acadêmicos da área da saúde da Faculdade Ciências da Vida.Revista Brasileira de Ciências da Vida, [S.I.], v. 5, n. 5, dez. 2017. ISSN 2525-359X. Disponível em: <http://jornal.faculdadecienciasdavida.com.br/index.php/RBCV/article/view/388>. Acesso em: 06 ago. 2018.

[2] Oliveira, Amanda A. de; Salvi, Jeferson de O.. Percepções de acadêmicos de farmácia sobre a homeopatia. Revista de homeopatia, São Paulo, v. 77, n. 1/2, p.16-20, dez. 2014. Disponível em: <http://revista.aph.org.br/index.php/aph/article/view/276/339>. Acesso em: 06 ago. 2018.

[3] ALTMAN, Max. 1755 - Nasce Hahnemann, médico criador da homeopatia. Abril 2015. Revista História Ciências Saúde Manguinhos, FIOCRUZ. Disponível em: <http://www.revistahcsm.coc.fiocruz.br/1755-nasce-hahnemann-medico-criador-da-homeopatia/>. Acesso em: 12 ago. 2018.

[4] BRASIL. ANVISA. . CONCEITOS E DEFINIÇÕES EM HOMEOPATIA. Disponível em: <http://www.anvisa.gov.br/hotsite/farmacopeiabrasileira/arquivos/cp38_2010/v_conceitos_definico es_homeopatia.pdf>. Acesso em: 10 ago. 2018.

[5] Souza Junior, E. A.. Souza, L. A.. Nunes, M. L. P.. Soares, T. M. P. Conhecimento e interesse dos acadêmicos médicos em medicina alternativa: acupuntura e homeopatia. Revista Científica Semana Acadêmica. Fortaleza, ano MMXV, №. 000072, 02/09/2015.

Disponível em: https://semanaacademica.org.br/artigo/conhecimento-e-interesse-dos-academicos- 
medicos-em-medicina-alternativa-acupuntura-e Acessado em: 06/08/2018.

[6] World Health Organization. WHO traditional medicine strategy 2002-2005. Geneva; 2002. Avaliable from: http://www.who.int/medicines/publications/traditionalpolicy/en/index.html Acessado em 25/08/2018.

[7] Loch-Neckel, Gecioni; Carmignan, Françoise; Crepaldi, Maria Aparecida. A homeopatia no SUS na perspectiva de estudantes da área da saúde. Rev. bras. educ. med., Rio de Janeiro, v. 34, n. 1, p. 82-90, Mar. 2010. Available from

$<$ http://www.scielo.br/scielo.php?script=sci_arttext\&pid=S0100-

$55022010000100010 \& \operatorname{lng}=$ en\&nrm=iso $>$. access on 06 Aug. 2018.

http://dx.doi.org/10.1590/S0100-55022010000100010.

[8] Guerriero lara Coelho Zito. Approval of the Resolution governing the ethics of research in social sciences, the humanities, and other disciplines that use methodologies characteristic of these areas: challenges and achievements. Ciênc. saúde coletiva [Internet]. 2016 Aug [cited 2018 Aug 04] ; 21( 8 ): 2619-2629. Available from:

http://www.scielo.br/scielo.php?script=sci_arttext\&pid=S1413-81232016000802619\&Ing=en. http://dx.doi.org/10.1590/1413-81232015218.17212016.

[9] Galhardi W, Barros N, Leite-Mor A. A homeopatia na rede pública do Estado de São Paulo: facilitadores e dificultadores. Revista Brasileira de Medicina de Família e

Comunidade [Internet]. 2012 Mar 26; [Citado em 2018 Ago 25]; 7(22): 35-43. Disponível em: https://www.rbmfc.org.br/rbmfc/article/view/413

[10] Furukawa, Cristiane; Morikawa, Tânia; Gutiérrez, Márcia A.. Pesquisa sobre o Conhecimento e Interesse no Tratamento Homeopático em uma Amostra da População de São Caetano do Sul. Cultura Homeopática, São Paulo, v. 18, n. 10, p.10-12, 22 fev. 2007. Trimestral.

[11] Souza, M. L. et al. Interesse em Homeopatia entre Estudantes da Faculdade de Ciências Médicas da Universidade Estadual de Campinas - SP. Revista Brasileira de Homeopatia, São Paulo, v. 2, n. 7, p.14-20, dez. 2001. Disponível em: <http://www.ihb.org.br/br/docs/revista/v.7.n.2 2001/pdf/p.14-20.pdf>. Acesso em: 06 ago. 2018.

[12] Matos, Roseana Maria de Araújo. A PRODUÇÃO DO CONHECIMENTO EM HOMEOPATIA E SEU ENSINO NAS FACULDADES DE MEDICINA DAS UNIVERSIDADES FEDERAIS

BRASILEIRAS. 2009. 106 f. Dissertação (Mestrado) - Curso de Programa de Pós-graduação em Educação em Ciências e Saúde, Centro de Ciências da Saúde, Universidade Federal do Rio de Janeiro, Rio de Janeiro, 2009. Disponível em:

<http://www.nutes.ufrj.br/mestrado/arquivos/dis.roseana.pdf>. Acesso em: 06 ago. 2018.

[13] LOCH-NECKEL, Gecioni; CARMIGNAN, Françoise; CREPALDI, Maria Aparecida. A homeopatia no SUS na perspectiva de estudantes da área da saúde. Rev. bras. educ. med., Rio de Janeiro, v. 34, n. 1, p. 82-90, Mar. 2010. Available from

$<$ http://www.scielo.br/scielo.php?script=sci_arttext\&pid=S0100-

$55022010000100010 \& \operatorname{lng}=$ en\&nrm=iso $>$. access

on 25 Aug. 2018. http://dx.doi.org/10.1590/S0100-55022010000100010.

[14] Teixeira, Marcus Zulian. Homeopatia: desinformação e preconceito no ensino médico. Rev. bras. educ. med., Rio de Janeiro, v. 31, n. 1, p. 15-20, Apr. 2007 . Available from $<$ http://www.scielo.br/scielo.php?script=sci_arttext\&pid=S0100-

$55022007000100003 \& \operatorname{lng}=e n \& n r m=i s 0>$. access

on 25 Aug. 2018. http://dx.doi.org/10.1590/S0100-55022007000100003.

[15] MARCUS ZULIAN TEIXEIRA. Associação Paulista de Homeopatia. Editorial: Aos que clamam pelas evidências científicas em homeopatia. Revista de Homeopatia, São Paulo, v. 80, n. 1/2, p.01-03, 2017. Semestral. 

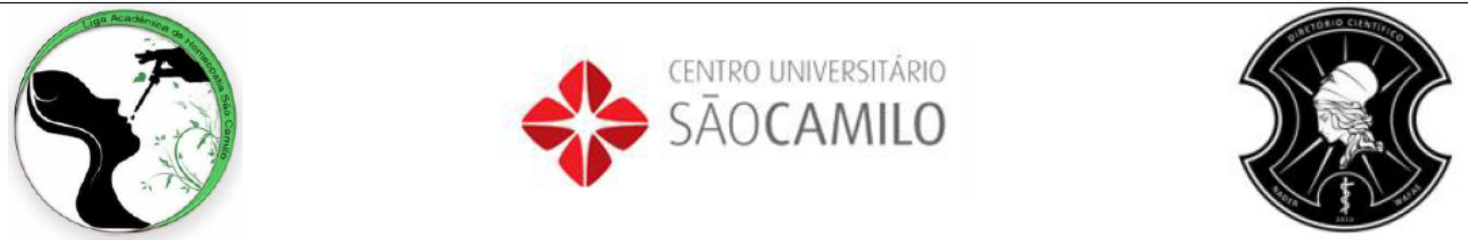

\section{Questionário: "Conhecimentos básicos da população sobre Homeopatia"}

I. Identificação:

1. Sexo:
( ) Feminino
( ) Masculino

2. Faixa etária:
( ) 15 a 20 anos;
( ) 21 a 30 anos;
( ) 31 a 40 anos;
( ) 41 a 50 anos;
( ) Acima de 50 anos.

3. Escolaridade:

Fundamental

( ) incompleto ( ) completo

Médio

( ) incompleto ( ) completo

Universitário

( ) incompleto ( ) completo

II. De acordo com o seu conhecimento, escolha a (s) melhor (es) alternativa (s) para cada questão:

1. Você já ouviu falar em Homeopatia?
( ) Sim
( ) Não

2. Se sim, você teve conhecimento sobre Homeopatia:

( ) Através de um médico;

( ) Através de livros, jornais, revistas ou televisão;

( ) Através de um amigo, vizinho ou parente;

( ) Em uma farmácia.
3. Você já fez tratamento com Homeopatia?
( ) Sim ( ) Não

4. Para você, Homeopatia é:

( ) Uma especialidade da área da saúde que utiliza medicamentos para tratar doenças;

( ) Uma terapia com psicólogo;

( ) Uma prática religiosa;

( ) Não sei.

5. Você acha que no tratamento homeopático são utilizados:

( ) Apenas produtos derivados de plantas;

( ) Agulhas;

( ) Medicamentos preparados em glóbulos ("bolinhas") e gotas;

( ) Não sei.

6. Você acha que o médico homeopata é o especialista que cuida de:

( ) Apenas doenças do sangue;

( ) Apenas doenças alérgicas;

( ) Doenças físicas e doenças de causa emocional, tratando do indivíduo como um todo;

( ) Não sei.

"Pesquisa sobre o Conhecimento e Interesse no Tratamento Homeopático em uma Amostra da População de São Caetano do Sul".

Autores: Cristiane Furukawa, Tânia Murikawa, Márcia A. Gutiérrez, 2007 


\section{ANEXO 2: PANFLETO INFORMATIVO}

\section{O que é Homeopatia?}

É um tipo de tratamento que utiliza produtos evtraidos de plantas, minerais ou animais.

Os remédios são preparados em gotas ou em glóbulos ("bolinhas") com as substâncias diluidas em água.

Chús e produtos naturais NAT̃O são Homeopatia!

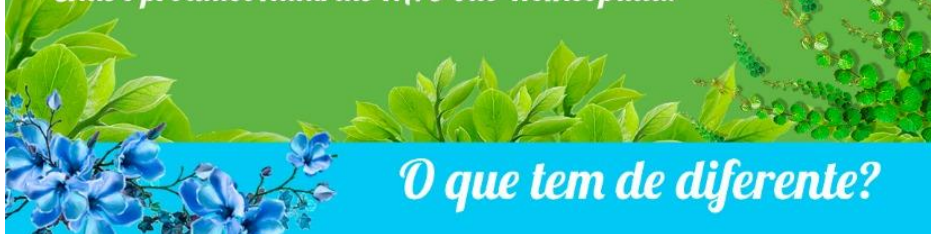

Na consultia homeopática são levados em consideração sintomas físicos e sintomas emocionais do paciente.

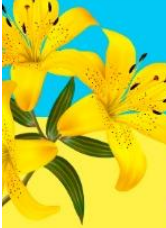

\section{Quem pode se tratar com homeopatia?}

Qualquer pessoa, de qualquer idade: recém-nascidos, crianças, adultos, gestantes e idosos.

Quem está usando medicamentos convencionais para outras doenças também pode fazer uso de medicamentos homeopáticos ao mesmo tempo.

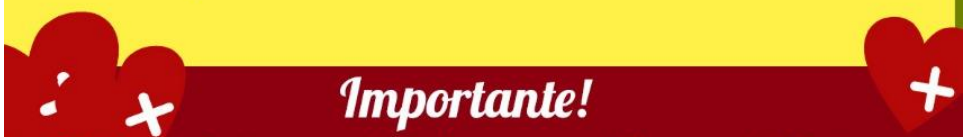

Otratamento deve ser orientado por um médico especialista em

Homeopatia.

Você pode agendar uma consulta com médico homeopata na rede pública de saíde pelo SUS! 